OPEN ACCESS

Edited by:

Nanyin Zhang

Pennsylvania State University,

United States

Reviewed by:

Yu-Feng Zang,

Hangzhou Normal University, China

Zhi Yang,

Shanghai Mental Health Center

(SMHC), China

*Correspondence:

Yi Shao

freebee99@163.com

${ }^{\dagger}$ These authors have contributed equally to this work

Specialty section: This article was submitted to Brain Imaging Methods, a section of the journal Frontiers in Neuroscience

Received: 09 August 2018 Accepted: 05 July 2019

Published: 23 July 2019

Citation:

Xu M-W, Liu H-M, Tan G, Su T, Xiang $C-Q, W u W, L i B, L i n$, Xu X-W, Min Y-L, Liu W-f, Gao G-P and Shao Y (2019) Altered Regional Homogeneity in Patients With Corneal Ulcer: A Resting-State Functional MRI Study. Front. Neurosci. 13:743 doi: 10.3389/fnins.2019.00743

\section{Altered Regional Homogeneity in Patients With Corneal Ulcer: A Resting-State Functional MRI Study}

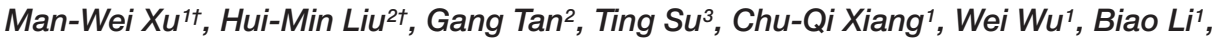 \\ Qi Lin', Xiao-Wei Xu1, You-Lan Min'1, Wen-feng Liu', Gui-Ping Gao' ${ }^{1}$ and Yi Shao'*
}

${ }^{1}$ Department of Ophthalmology, The First Affiliated Hospital of Nanchang University, Jiangxi Province Ocular Disease Clinical Research Center, Nanchang, China, ${ }^{2}$ Department of Ophthalmology, University of South China, Hengyang, China, ${ }^{3}$ Eye Institute of Xiamen University, Fujian Provincial Key Laboratory of Ophthalmology and Visual Science, Xiamen, China

Objective: To investigate the potential regional homogeneity (ReHo) brain activity changes in patients with corneal ulcer $(\mathrm{CU})$ and their possible relationship with clinical symptoms.

Materials and Methods: Forty patients with CU (26 men and 14 women), and 40 healthy controls ( $\mathrm{HCs}$ ) (26 men and 14 women) closely matched in age, sex, and weight underwent resting-state functional MRI scans, respectively. The ReHo method was applied to evaluate synchronous neural activity changes. Receiver operating characteristic curve (ROC curve) was used to show high test-retest stability and high degree of sensitivity and specificity. We utilized the correlation analysis to calculate the relationship between the average ReHo signal values in different brain areas and the clinical symptoms in CU patients.

Results: Compared with the HCs, CU patients had significantly increased ReHo values in right cerebellum posterior lobe, left cerebellum posterior lobe, left inferior temporal gyrus, right lingual gyrus, left middle frontal gyrus, left angular gyrus, left cingulate gyrus, right angular gyrus and bilateral superior frontal gyrus, and decreased ReHo values in right anterior cingulate and left precentral gyrus. ROC curve analysis of each brain regions showed the accuracy of AUC was perfect except the right cerebellum posterior lobe. Nevertheless, there was no clear evidence of prominent relevance between the average ReHo values in brain areas and the clinical symptoms.

Conclusion: Corneal ulcer caused dysfunctional adaption in different brain areas, which including relatively increased values and decreased values. This finding may help us take a further step in exploring the underlying pathologic mechanisms of $\mathrm{CU}$.

Keywords: corneal ulcer, regional homogeneity, functional MRI, resting state, brain activity

\section{INTRODUCTION}

Corneal ulcer (CU), a common ophthalmological disease, is a kind of inflammatory reaction of cornea. In worldwide, corneal disease is the fifth leading cause of blindness, following cataract, refractive error, glaucoma, and age related macular degeneration (Flaxman et al., 2017). According to etiology, CU can be divided into infectious $\mathrm{CU}$ and non-infectious $\mathrm{CU}$ and in China, 
the infectious factors of virus, fungus, bacteria, and acanthamoeba, plays the leading role in resulting in $\mathrm{CU}$ (Song et al., 2011). The morbidity of infectious CU is $0.148 \%$ in middle-China (Langkide et al., 2002).

Common symptoms of CU are massive pain, blepharospasm, photophobia, and tearing (Herz et al., 2008). If diagnose delayed, CU can result in severe complications including corneal perforation, endophthalmitis, and iris atrophy (Herz et al., 2008; Rana et al., 2015; Zapp et al., 2018). The tentative diagnosis of CU can often be made by slit lamp, fluorescein staining and in vivo confocal microscopy (IVCM) (Herz et al., 2008). A previous research already showed that corneal disease had influence on brain area activities (Arrigo et al., 2018). Nevertheless, the inspection methods mentioned above only focused on the effects of the ocular surface on brain activities and ignored the rest of the visual system containing the eye and the connecting pathways through the visual cortex and other regions of brain.

Synchronous neuronal activity occurs in the normal human brain (Sturm and König, 2001). Synchronous neuronal activity plays a critical role in learning and memory (Jutras and Buffalo, 2010). In addition, reliable propagation of synchronous neuronal activity was shown to be crucial for neuronal information processing (Bayati et al., 2015). Several previous electroencephalographic and functional magnetic resonance imaging (fMRI) studies indicated that synchronous neuronal activity might have a critical role in neurophysiological activity (Ward, 2003; Spencer et al., 2004; Li et al., 2015). The regional homogeneity ( $\mathrm{ReHo}$ ) is widely used to investigate the local synchronization of spontaneous fMRI signals. It has been successfully applied to previous researches about evaluating the brain activities in patients with ocular diseases (Cui et al., 2014; Song et al., 2014; Shao et al., 2015; Huang et al., 2016a,b, 2017a,b). Our study was to determine whether the $\mathrm{CU}$ patients were associated with abnormal synchronous neuronal activity related to eye pain perception and visual loss.

\section{MATERIALS AND METHODS}

\section{Subjects}

The Ophthalmology Department of the First Affiliated Hospital of Nanchang University enlisted $40 \mathrm{CU}$ patients (26 men and 14 women). The inclusion criteria of the study in CU patients were (1) the duration of CU was at least 14 days; and (2) there were no other ocular diseases in any of bilateral eyes (cataracts, glaucoma, retinal degeneration, amblyopia, strabismus, optic neuritis, etc.).

Corneal ulcer with the following conditions were excluded from the study: (1) impending perforation, corneal perforation and blindness; (2) ocular trauma; (3) systemic diseases including hypertension and heart disease; (4) psychiatric diseases; and (5) other disorders that would affect the ReHo measurement.

Totally 40 healthy controls (HCs, 26 men and 14 women) participated in the study and had no statistic differences in age, sex and weight level with patients of CU group. All the HCs met the following criteria: (1) no neurological or psychiatric disorders (Parkinson's disease, manic depression, depressive disorder and so on); and (2) be qualified to undergo MRI scanning (e.g., no metallic false teeth, cardiac pacemaker or other implanted metal devices, and so on).

The experiment was approved by the First Affiliated Hospital of Nanchang ethics committee. All the methods applied in the research followed the Declaration of Helsinki and complied with the medical ethics. For each subject, the research pact and process were fully explained, and written agreement was acquired.

\section{MRI Parameters}

Magnetic resonance imaging scanning was carried out on a 3-T MR scanner (Trio, Siemens, Munich, Germany). High-resolution $T_{1}$-weighted images were obtained with a three-dimensional spoiled gradient-recalled sequence, using the following parameters: repetition time $=1900 \mathrm{~ms}$, echo time $=2.26 \mathrm{~ms}$, thickness $=1.0 \mathrm{~mm}$, gap $=0.5 \mathrm{~mm}$, acquisition matrix $=256 \times 256$, field of view $=250 \mathrm{~mm} \times 250 \mathrm{~mm}$, and flip angle $=9^{\circ}$. Functional images with the parameters (repetition time $=2,000 \mathrm{~ms}$, echo time $=30 \mathrm{~ms}$, thickness $=4.0 \mathrm{~mm}$, gap $=1.2 \mathrm{~mm}$, acquisition matrix $=64 \times 64$, flip angle $=90^{\circ}$, field of view $=220 \mathrm{~mm} \times 220 \mathrm{~mm}, 29$ axial) were corrected.

\section{fMRI Data Analysis}

First, all the images were checked by MRIcro $^{1}$ to delete deficient ones. Then we preprocessed the rest of the images by $\mathrm{SPM}^{2}$ and DPARSF${ }^{3}$ software. Because there was time for the participants get used to the starting noise, we discarded the first 10 volumes of each subject. Then, applying slice timing, head motion correction (within $1.5 \mathrm{~mm}$ or $1.5^{\circ}$ in any of six parameters) and spatial normalization to the data. The next step is smoothening the data with a Gaussian kernel of $6 \mathrm{~mm}^{3} \times 6 \mathrm{~mm}^{3} \times 6 \mathrm{~mm}^{3}$ full width at halfmaximum (FWHM). Finally, with the echo-planar imaging template, the fMRI images were spatially standardized to the Montreal Neurological Institute (MNI) space and resampled at a resolution ratio of $3 \mathrm{~mm} \times 3 \mathrm{~mm} \times 3 \mathrm{~mm}$. After all the steps above, for reducing the effects of low-frequency drift, physiological high-frequency respiratory and cardiac noise, the fMRI data were detrended and bandpass-filtered (0.01$0.08 \mathrm{~Hz}$ ) finally.

Based on Kendall's coefficient of concordance (KCC), the data of ReHo values was dealt with $\mathrm{REST}^{4}$ software to analyze the correlation. The KCC can give voxels through calculating the KCC of time series of the voxel and nearest-neighbor ones (26 voxels): $\mathrm{W}=\sum\left(\mathrm{R}_{\mathrm{i}}\right)^{2}-\mathrm{n}(\mathrm{R})^{2} 2 \mathrm{~K}^{2}\left(\mathrm{n}^{3}-\mathrm{n}\right) / 12$, where $\mathrm{W}$ represents the KCC among given voxels, ranging from 0 to 1 ; $\mathrm{R}_{i}$ represents the sum rank of the time point; $\mathrm{R}=(\mathrm{n}+1) \mathrm{K} / 2$ is the mean of the $\mathrm{R}_{i} s K$ is the number of time series within a measured cluster (in the study, $K=27$, plus one voxel located in the cubic center); $\mathrm{n}$ is the number of ranks. There, all the ReHo data were generated.

\footnotetext{
${ }^{1}$ www.MRIcro.com

${ }^{2}$ http://www.fil.ion.ucl.ac.uk/spm

${ }^{3}$ http://rfmri.org/DPARSF

${ }^{4}$ http://www.resting-fmri.Sourceforge.net
} 


\section{Statistical Analysis}

The fMRI data were examined by two-sample $t$-test with the SPM 8 software (two-tailed, voxel-level: $P<0.01$, GRF correction, cluster-level: $P<0.05)$ and it helps to compare two group differences in the zReHo maps using the GRF method which was used to correct for multiple comparisons and regressed covariates of age and sex.

\section{Brain-Behavior Correlation Analysis}

According to the ReHo calculation result, some different brain regions showed different signals between CU groups and HCs. For each region, the mean ReHo values was calculated by averaging over all voxels. The relationship between the mean ReHo value and their clinical features was calculated using the correlation analysis (the threshold was set at $0.001, P<0.001$ was considered statistically significant).

\section{Clinical Data Analysis}

All the accumulated clinical data were collected, including the course of the disease and the best-corrected visual acuity. The demographic and clinical variables between $\mathrm{CU}$ and $\mathrm{HC}$ groups were compared using SPSS20.0 software (SPSS, Chicago, IL, United States) with independent sample $t$-test, and $p<0.05$ was considered to have statistical significance.

To show high test-retest stability and high degree of sensitivity and specificity, we applied the receiver operating characteristic (ROC) curve method.

\section{RESULTS}

\section{Demographics and Visual Measurements}

There were no obvious differences in weight $(P=0.892)$ and age $(P=0.824)$ between two groups. Details are shown in Table 1.

\section{ReHo Differences}

Compared with HCs, CU patients had significantly increased ReHo values in right cerebellum middle frontal gyrus, left angular gyrus, left cingulate gyrus, right angular gyrus and bilateral superior frontal gyrus and decreased $\mathrm{ReHo}$ values in left precentral gyrus and right anterior cingulate (Figures 1, 2 and Table 2).

TABLE 1 | Conditions of participants included in the study.

\begin{tabular}{lcccc}
\hline Condition & CUs & HCs & $\boldsymbol{t}$ & $\boldsymbol{P}_{\text {-value* }}$ \\
\hline Male/female & $26 / 14$ & $26 / 14$ & $\mathrm{~N} / \mathrm{A}$ & $>0.99$ \\
Age (years) & $51.25 \pm 5.46$ & $51.98 \pm 5.18$ & 0.251 & 0.824 \\
Weight (kg) & $63.12 \pm 7.35$ & $63.89 \pm 6.73$ & 0.181 & 0.892 \\
Handedness & $40 \mathrm{R}$ & $40 \mathrm{R}$ & $\mathrm{N} / \mathrm{A}$ & $>0.99$ \\
Duration of CU (days) & $25.75 \pm 5.65$ & $\mathrm{~N} / \mathrm{A}$ & $\mathrm{N} / \mathrm{A}$ & $\mathrm{N} / \mathrm{A}$
\end{tabular}

${ }^{*} P<0.05$ Independent t-tests comparing two groups. HCs, healthy controls; N/A, not applicable; $\mathrm{CU}$, corneal ulcer.

\section{Receiver Operating Characteristic Curve}

To test whether the distinctive ReHo values detected from the $\mathrm{CU}$ and HC groups could differ CU patients from HCs, we performed the ROC curve analysis. The areas under the ROC curve (AUCs) for ReHo values were as follows: right cerebellum posterior lobe, 0.690; left cerebellum posterior lobe, 0.750; right cerebellum posterior lobe, 0.732 ; left inferior temporal gyrus, 0.726; right lingual gyrus, 0.789 ; left middle frontal gyrus, 0.774 ; left angular gyrus, 0.776; left cingulate gyrus, posterior lobe, left cerebellum posterior lobe, left inferior temporal gyrus, right lingual gyrus, left posterior lobe, left cerebellum posterior lobe, left inferior temporal gyrus, right lingual gyrus, left 0.805; right angular gyrus, 0.817 and bilateral superior frontal gyrus, 0.792 (CUs > HCs) (Figure 3A); left precentral gyrus, 0.757, and right anterior cingulate, 0.788 (CUs $<\mathrm{HCs}$ ) (Figure 3B).

\section{DISCUSSION}

This ReHo method has been successfully applied in several ophthalmological diseases and predicts huge development prospect (Table 3). As far as we know, this is the first study that the ReHo method has been used to estimate the effect of $\mathrm{CU}$ on resting-state brain activity till now. Compared to HCs, CU patients had significantly increased ReHo values in right cerebellum posterior lobe, left cerebellum posterior lobe, left inferior temporal gyrus, right lingual gyrus, left middle frontal gyrus, left angular gyrus, left cingulate gyrus, right angular gyrus and bilateral superior frontal gyrus, and decreased ReHo values in left precentral gyrus and right anterior cingulate (Figure 4).

\section{Analysis of the Increased ReHo Values in the CUs}

Cerebellum posterior lobe (CPL), the portion of the cerebellum below the primary fissure, is the main motor control area. Previous research shows that CPL is associated with primary insomnia (Li P. et al., 2018), cognitive vulnerability to depression (Sun et al., 2018), and depressive disorder (Zhang Y. et al., 2018). In our study, the CU patients had increased ReHo values in left and right CPL, it may explain that sleeping dysfunction and depressive symptom sometimes occurred to the CU patients. Besides, Like CPL, many studies have shown that people with depression have dysfunction in the middle frontal gyrus (MFG) (Chang et al., 2011; Nelson et al., 2017). The MFG lies between the inferior frontal sulcus and the superior frontal sulcus, behind the precentral sulcus. This area is responsible for human cognitive and attention function (Martin et al., 2009). Our results showed that spontaneous neuronal activity was increased in MGF, it may also contribute to the depression in CU patients. Emotional disturbance in CU patients also includes dysphoria, schizophrenia and other unusual mental states, these disorders may relate to abnormal activities of lingual gyrus (LG), cingulate gyrus (CG), and superior frontal gyrus (SFG) in CU patients. LG is located between the sulcus of the callosum and the posterior part of the collateral sulcus. This region is believed to play a tremendously important role in vision and dreaming. Hypermetabolism in the region has been 

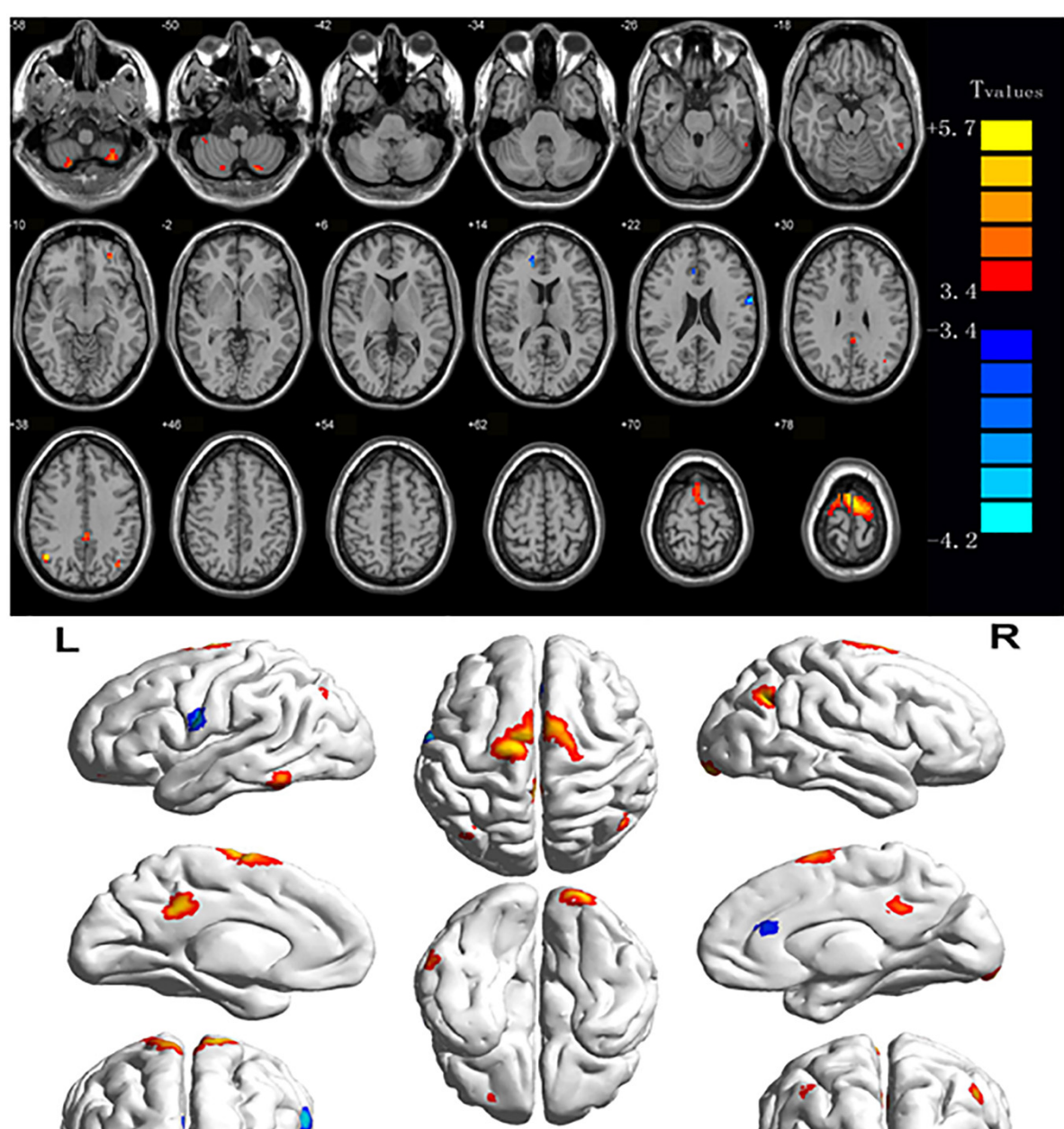

$\mathbf{R}$
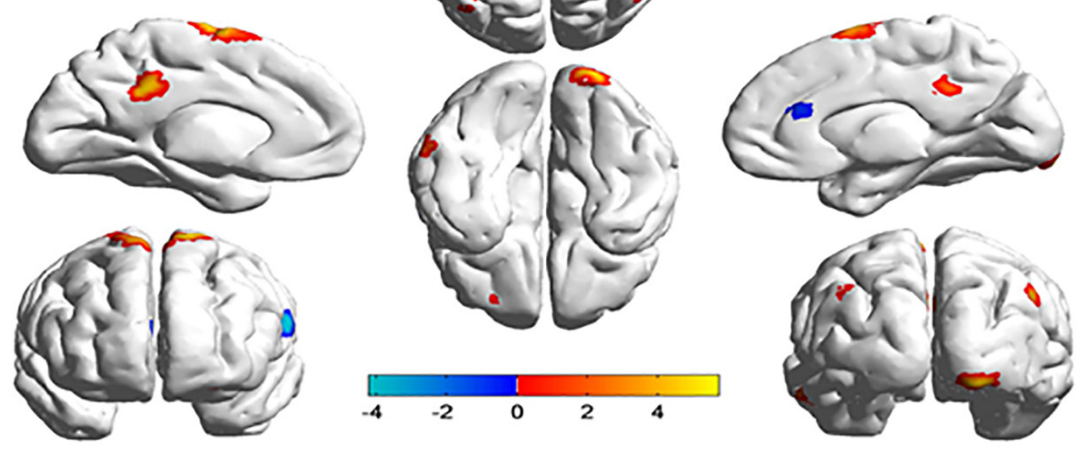

FIGURE 1 | Significant differences in ReHo values between the CU group and HCs.

associated with visual snow syndrome and headache (Schankin et al., 2014). There were researches showed that LG activity changes dramatically in schizophrenia (Chen, 2018; Zhang P. et al., 2018). In our study, the CU patients had increased ReHo values in right lingual gyrus, which may explain the symptoms of headache and dysphoria happened in CU patients. CG is an important component of the limbic system. It can be divided into anterior cingulate cortex (AC) and posterior cingulate, two totally different areas of different functions. The former one is involved in many complex bodily, visceral motor functions and pain responses, and the later one monitors sensory and stereoscopic positioning and memory functions. Previous study has shown that the metabolism of CG can affect the emotional disorder (Shukla et al., 2018). In our study, the CU patients had increased ReHo values in left CG but decreased ReHo values in right AC, the mechanism still remains exploring. SFG, a part of the middle frontal gyrus. Associated with deliberative decision making, SFG has significantly and positively abnormal fMRI signals in individuals under acute social stress (Chang and Yu, 2018). It also has relationship with cognitive function (Zheng J. et al., 2018). In our study, the CU patients had increased ReHo values in bilateral SFC, it may help explain the unusual mental state of CU patient. Speaking of cognitive function, the CU patients had increased ReHo values in left inferior temporal gyrus (ITG) in our study. ITG, also known as gyrus temporalis inferior, lies in the convolution or protuberance of temporal lobe of the cerebral hemispheres, which is beneath the middle temporal sulcus and the ITG region stretches to the inferior sulcus. This area is known to be associated with behavioral learning and object memory based on current research. It is also responsible for visual perception, involved in facial recognition and believed to be associated with cognitive processes (Tu et al., 2018; Zheng W. et al., 2018). Therefore, apart from the cornea factor, the abnormal ITG and 


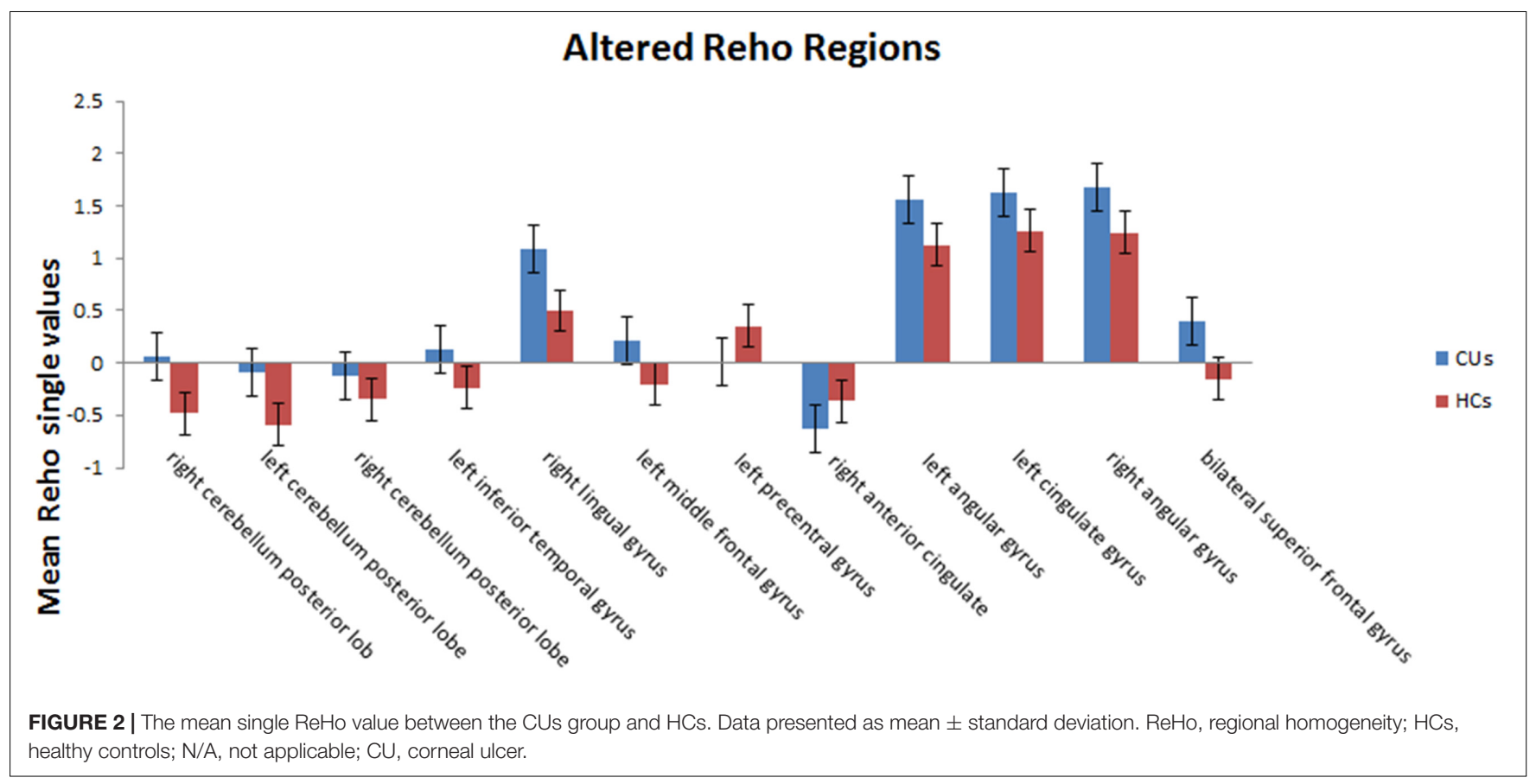

TABLE 2 | Brain regions with significantly different ReHo values between the CUs and HCs.

\begin{tabular}{|c|c|c|c|c|c|c|c|c|}
\hline \multirow[t]{2}{*}{ Condition } & \multirow[t]{2}{*}{ Left/right } & \multirow[t]{2}{*}{ Brain areas } & \multirow[t]{2}{*}{ BA voxels } & \multicolumn{3}{|c|}{ MNI coordinates } & \multirow[t]{2}{*}{ Peak } & \multirow[t]{2}{*}{$T$-value } \\
\hline & & & & $x$ & $Y$ & $z$ & & \\
\hline 1 & Right & Cerebellum posterior lobe & / & 18 & -69 & -57 & 21 & 4.2291 \\
\hline 2 & Left & Cerebellum posterior lobe & / & -33 & -63 & -57 & 30 & 4.497 \\
\hline 3 & Right & Cerebellum posterior lobe & / & 39 & -45 & -48 & 15 & 4.0563 \\
\hline 6 & Left & Middle frontal gyrus & 11 & -27 & 48 & -6 & 16 & 4.2676 \\
\hline 7 & Left & Angular gyrus & 39 & -39 & -69 & 36 & 21 & 4.6547 \\
\hline 8 & Left & Cingulate gyrus & 32 & -3 & -42 & 33 & 36 & 4.8645 \\
\hline 9 & Right & Angular gyrus & 39 & 48 & -63 & 39 & 22 & 5.742 \\
\hline 10 & / & Bilateral superior frontal gyrus & 31 & -3 & -3 & 78 & 275 & 5.7209 \\
\hline
\end{tabular}

Voxel-level: $P<0.01$, GRF correction, cluster-level: $P<0.05$. CU, corneal ulcer; HCs, healthy controls; BA, Brodmann area; MNI, Montreal Neurological Institute.

SFC activities may contribute to obstacle of item identification, too. Angular gyrus (AG) is an important associative region in the back of the brain above the Wernicke region and at the apex occipital lobe. It transfers visual information to Wernicke's area, and understands the meanings of perceived words visually (Hall, 2010). It is also involves a number of processes associated with digital processing, language learning, spatial cognition, memory retrieval, and psychological theory. Previous research showed that attention-deficit was associated with abnormal AG activities (Kong et al., 2017). In our study, the $\mathrm{CU}$ patients had increased ReHo values in left and right
AG, which may suggest that dysfunction of attention showed in $\mathrm{CU}$ is reasonable.

\section{Analysis of the Decreased ReHo Values in the $\mathrm{CU}$}

Precentral gyrus (PG), or anterior central gyrus, mostly lies on the lateral (convex) side of the cerebral hemispheres. Its borders are the precentral sulcus above; and the lateral fissure below. PG is the cortical motor area, and manages the opposite side half body to move at will. According to recent researches, this 
A

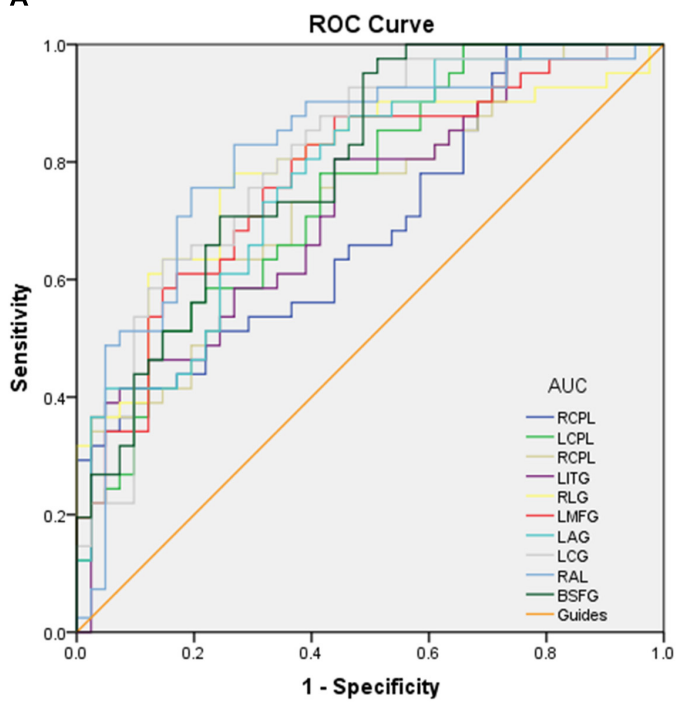

B

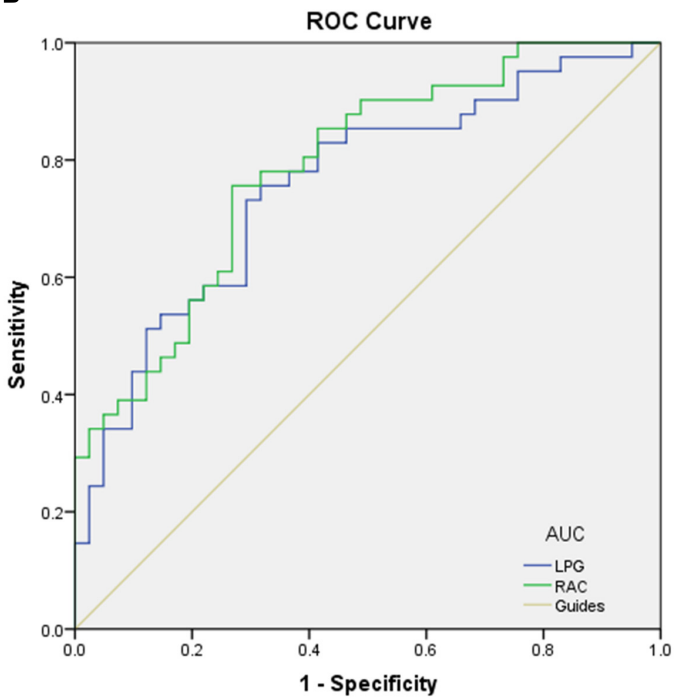

FIGURE 3 | Receiver operating characteristic curve analysis of the ReHo values for altered brain regions. (A) The area under the ROC curve were RCPL 0.690 $(p=0.003 ; 95 \%$ Cl: 0.577-0.803), LCPL 0.750 ( $p<0.001 ; 95 \%$ Cl: 0.646-0.853), RCPL 0.732 ( $p<0.001 ; 95 \%$ Cl: 0.624-0.839), LITG 0.726 ( $<$ < $0.001 ; 95 \%$ Cl: 0.618-0.835), RLG 0.789 ( $p<0.001 ; 95 \%$ Cl: 0.689-0.890), LMFG 0.774 ( $p<0.001 ; 95 \%$ Cl: 0.673-0.875), LAG 0.776 ( $p<0.001 ; 95 \%$ Cl: $0.677-0.875)$, LCG 0.805 ( $p<0.001 ; 95 \%$ Cl: 0.710-0.901), RAL 0.817 ( $p<0.001 ; 95 \%$ Cl: 0.722-0.913), and BSFG 0.792 ( $p<0.001 ; 95 \%$ Cl: $0.697-0.888)$. (B) The area under the ROC curve were LPG $0.757(p<0.001 ; 95 \%$ Cl: 0.652-0.861) and RAC 0.788 ( $<0.001$; 95\% Cl: 0.691-0.884). ROC, receiver operating characteristic; ReHo, regional homogeneity; RCPL, right cerebellum posterior lobe; LCPL, left cerebellum posterior lobe; LITG, left inferior temporal gyrus; RLG, right lingual gyrus; $L M F G$, left middle frontal gyrus; LAG, left angular gyrus; LCG, left cingulate gyrus; RAL, right angular gyrus; BSFG, bilateral superior frontal gyrus; LPG, left precentral gyrus; RAC, right anterior cingulate.

TABLE 3 | ReHo method applied in ophthalmological diseases.

\begin{tabular}{ll}
\hline References & Disease \\
\hline Song et al., 2014 & Glaucoma \\
Shao et al., 2015 & Optic neuritis \\
Huang et al., 2016b & Strabismus \\
Cui et al., 2014 & Diabetic retinopathy \\
Huang et al., 2016a & Open-globe injury \\
Huang et al., 2017b & Late monocular blindness \\
Huang et al., 2017a & Retinal detachment \\
\hline
\end{tabular}

area also has relationship with depressive disorder ( $\mathrm{Li} \mathrm{C}$. et al., 2018) and memory performance (Shang et al., 2018). In our study, the CU patients had decreased ReHo values in left PG, which may implicit the poor memory and black mood happened in CU. And the finding may explain the skin paresthesia of some CU patients.

Receiver operating characteristic curve proves the reliability of the results. The accuracy is perceived as perfect when AUC values is $0.7-0.9$, a value between 0.5 and 0.7 is considered moderate, and less than 0.5 means the discrimination result is low. The ROC curve analysis in our study showed that the AUCs of each brain regions were over 0.7 , which might represent these specific ReHo differences have a proper diagnostic accuracy in identifying CU. In brief, our findings demonstrated that the ReHo method might be a sensitive measurement of fMRI to diagnose CU patients in the future.

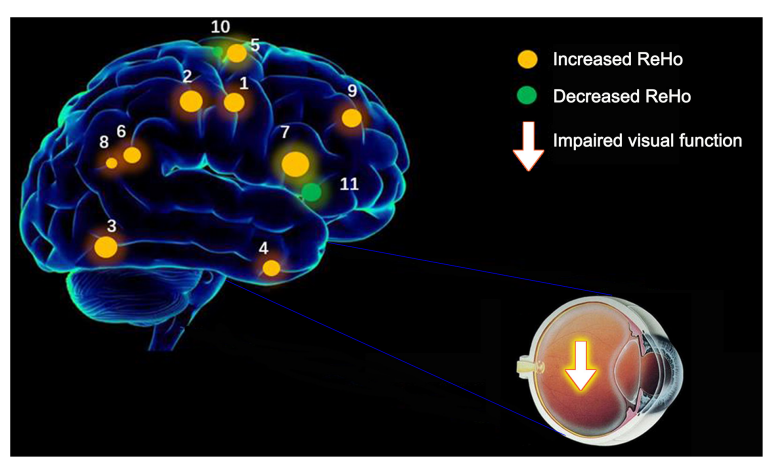

FIGURE 4 | The ReHo results of brain activity in the CU group. Compared with the HCs, the ReHo of the following regions were decreased to various extents: 1-right cerebellum posterior lobe $(t=4.2291)$, 2-left cerebellum posterior lobe $(t=4.497)$, 3-left inferior temporal gyrus $(t=3.8959)$, 4-right lingual gyrus $(t=4,9238), 5$-left middle frontal gyrus $(t=4.2676)$, 6-left angular gyrus ( $t=4.6547)$, 7 -left cingulate gyrus $(t=4.8645)$, 8 -right angular gyrus $(t=5.742)$, 9-bilateral superior frontal gyrus $(t=5.7209)$, 10-left precentral gyrus ( $t=-4.1336)$, and 11-right anterior cingulate $(t=-4.2228)$. The sizes of the spots denote the degree of quantitative changes. ReHo, regional homogeneity; HCs, healthy controls; CU, corneal ulcer.

\section{CONCLUSION}

In summary, the results showed that patients with $\mathrm{CU}$ had abnormal brain changes, performed as spontaneous activity. These findings provide important information for the 
understanding and lay a foundation of further study of the neural mechanisms underlying CU.

\section{ETHICS STATEMENT}

The experiments were approved by the First Affiliated Hospital of Nanchang ethics committee. All the methods applied in the research followed the Declaration of Helsinki and complied with the medical ethics. For each subject, the research pact and process were fully explained, and written agreement was acquired.

\section{AUTHOR CONTRIBUTIONS}

YS contributed to the conception and design of the study. GT, C-QX, WW, and G-PG organized the database. TS, BL, X-WX,

\section{REFERENCES}

Arrigo, A., Rania, L., Calamuneri, A., Postorino, E., Mormina, E., Gaeta, M., et al. (2018). Early corneal innervation and trigeminal alterations in parkinson disease: a pilot study. Cornea 37, 448-454. doi: 10.1097/ICO. 0000000000001517

Bayati, M., Valizadeh, A., Abbassian, A., and Sen, C. (2015). Self-organization of synchronous activity propagation in neuronal networks driven by local excitation. Front. Comput. Neurosci. 9:69. doi: 10.3389/fncom.2015.00069

Chang, C. C., Yu, S. C., Mcquoid, D. R., Messer, D. F., Taylor, W. D., Singh, K., et al. (2011). Reduction of dorsolateral prefrontal cortex gray matter in late-life depression. Psychiatry Res. Neuroimaging 193, 1-6. doi: 10.1016/j.pscychresns. 2011.01.003

Chang, J., and Yu, R. (2018). Acute social stress modulates coherence regional homogeneity. Brain Imaging Behav. 13, 762-770. doi: 10.1007/s11682-0189898-9

Chen, B. (2018). Abnormal regions in functional connectivity of chronic schizophrenia. Int. J. Neurosci. 128, 1150-1156. doi: 10.1080/00207454.2018. 1486836

Cui, Y., Jiao, Y., Chen, Y. C., Wang, K., Gao, B., Wen, S., et al. (2014). Altered spontaneous brain activity in type 2 diabetes: a resting-state functional MRI study. Diabetes 63, 749-760. doi: 10.2337/db13-0519

Flaxman, S. R., Bourne, R. R. A., Resnikoff, S., Ackland, P., Braithwaite, T., Cicinelli, M. V., et al. (2017). Global causes of blindness and distance vision impairment 1990-2020: a systematic review and meta-analysis. Lancet Glob. Health 5, e1221-e1234. doi: 10.1016/S2214-109X(17)30393-5

Hall, J. (2010). Guyton and Hall Textbook of Medical Physiology. Amsterdam: Elsevier, 699.

Herz, N. L., Matoba, A. Y., and Wilhelmus, K. R. (2008). Rapidly progressive cataract and iris atrophy during treatment of Acanthamoeba keratitis. Ophthalmology 115, 866-869. doi: 10.1016/j.ophtha.2007.05.054

Huang, X., Li, D., Li, H., Zhong, Y., Freeberg, S., Bao, J., et al. (2017a). Abnormal regional spontaneous neural activity in visual pathway in retinal detachment patients: a resting-state functional MRI study. Neuropsychiatr. Dis. Treat. 13, 2849-2854. doi: 10.2147/NDT.S147645

Huang, X., Ye, C., Zhong, Y., Ye, L., Yang, Q., Li, H., et al. (2017b). Altered regional homogeneity in patients with late monocular blindness: a restingstate functional MRI study. Neuroreport 28, 1085-1091. doi: 10.1097/WNR. 0000000000000855

Huang, X., Li, H. J., Ye, L., Zhang, Y., Wei, R., Zhong, Y. L., et al. (2016a). Altered regional homogeneity in patients with unilateral acute open-globe injury: a resting-state functional MRI study. Neuropsychiatr. Dis. Treat. 12, 1901-1906. doi: 10.2147/NDT.S110541

Huang, X., Li, S. H., Zhou, F. Q., Zhang, Y., Zhong, Y. L., Cai, F. Q., et al. (2016b). Altered intrinsic regional brain spontaneous activity in patients with comitant strabismus: a resting-state functional MRI study. Neuropsychiatr. Dis. Treat. 12, 1303-1308.
Y-LM, and W-fL carried out the statistical analysis. M-WX and H-ML wrote the first draft of the manuscript. All authors contributed to the manuscript revision, read, and approved its final version.

\section{FUNDING}

This study was supported by the National Natural Science Foundation of China (Nos. 81660158, 81160118, and 81400372); the Natural Science Key Project of Jiangxi Province (No. 20161ACB21017); the Youth Science Foundation of Jiangxi Province (No. 20151BAB215016); the Technology and Science Foundation of Jiangxi Province (No. 20151BBG70223); and the Health Development Planning Commission Science Foundation of Jiangxi Province (No. 20175116).

Jutras, M. J., and Buffalo, E. A. (2010). Synchronous neural activity and memory formation. Curr. Opin. Neurobiol. 20, 150-155. doi: 10.1016/j.conb.2010. 02.006

Kong, X., Xu, S., Sun, Y., Wang, K., Wang, C., Zhang, J., et al. (2017). Electroconvulsive therapy changes the regional resting state function measured by regional homogeneity ( $\mathrm{ReHo}$ ) and amplitude of low frequency fluctuations (ALFF) in elderly major depressive disorder patients: an exploratory study. Psychiatry Res. Neuroimaging 264, 13-21. doi: 10.1016/j.pscychresns.2017. 04.001

Langkide, A. R., Frederiksen, J. L., Rostrup, E., and Larsson, H. B. (2002). Functional MRI of the visual testing in patients with previous optic optic neuritis. Eur. J. Neurol. 9, 277-286. doi: 10.1046/j.1468-1331.2002.00399.x

Li, P., Ding, D., Ma, X., Zhang, H., Liu, J., and Zhang, M. (2018). Altered intrinsic brain activity and memory performance improvement in patients with endstage renal disease during a single dialysis session. Brain Imaging Behav. 12, 1640-1649. doi: 10.1007/s11682-018-9828-x

Li, C., Dong, M., Yin, Y., Hua, K., Fu, S., and Jiang, G. (2018). Aberrant effective connectivity of the right anterior insula in primary insomnia. Front. Neurol. 9:317. doi: 10.3389/fneur.2018.00317

Li, R., Li, Y., An, D., Gong, Q., Zhou, D., and Chen, H. (2015). Altered regional activity and inter-regional functional connectivity in psychogenic non-epileptic seizures. Sci. Rep. 5:11635. doi: 10.1038/srep11635

Martin, A., Martin, Y., Arvid, L., and Lundervold, A. J. (2009). Correlations between measures of executive attention and cortical thickness of left posterior middle frontal gyrus - a dichotic listening study. Behav. Brain Funct. 5, 41. doi: 10.1186/1744-9081-5-41

Nelson, J. D., Craig, J. P., Akpek, E. K., Caffery, B., Dua, H. S., Joo, C. K., et al. (2017). TFOS DEWS II introduction. Ocular Surf. 15, 269-275.

Rana, M., Lau, A., Aralikatti, A., and Shah, S. (2015). Severe microbial keratitis and associated perforation after corneal crosslinking for keratoconus. Cont. Lens Anterior Eye 38, 134-137. doi: 10.1016/j.clae.2014.10.004

Schankin, C. J., Maniyar, F. H., Sprenger, T., Chou, D. E., Eller, M., and Goadsby, P. J. (2014). The relation between migraine, typical migraine aura and "visual snow". Headache 54, 957-966. doi: 10.1111/head.12378

Shang, C., Lin, H., Tseng, W., and Gau, S. (2018). A haplotype of the dopamine transporter gene modulates regional homogeneity, gray matter volume, and visual memory in children with attention-deficit/hyperactivity disorder. Psychol. Med. 48, 2530-2540. doi: 10.1017/S003329171800 0144

Shao, Y., Cai, F. Q., Zhong, Y. L., Huang, X., Zhang, Y., Hu, P. H., et al. (2015). Altered intrinsic regional spontaneous brain activity in patients with optic neuritis: a resting-state functional magnetic resonance imaging study. Neuropsychiatr. Dis. Treat. 11, 3065-3073. doi: 10.2147/NDT.S92968

Shukla, D., Wijtenburg, S., Chen, H., Chiappelli, J., Kochunov, P., Hong, L., et al. (2018). Anterior cingulate glutamate and GABA associations on functional connectivity in schizophrenia. Schizophr. Bull. doi: 10.1093/schbul/sby075 [Epub ahead of print]. 
Song, X. S., Tian, L., and Xie, L. X. (2011). [Infectious keratitis in China during the past two decades: a bibliometric analysis]. Zhonghua Yi Xue Za Zhi. 91, 1104-1107.

Song, Y., Mu, K., Wang, J., Lin, F., Chen, Z., Yan, X., et al. (2014). Altered spontaneous brain activity in primary open angle glaucoma: a resting-state functional magnetic resonance imaging study. PLoS One 9:e89493. doi: 10.1371/ journal.pone.0089493

Spencer, K. M., Nestor, P. G., Perlmutter, R., Niznikiewicz, M. A., Klump, M. C., Frumin, M., et al. (2004). Neural synchrony indexes disordered perception and cognition in schizophrenia. Proc. Natl. Acad. Sci. U.S.A. 101, 17288-17293. doi: 10.1073/pnas.0406074101

Sturm, A. K., and König, P. (2001). Mechanisms to synchronize neuronal activity. Biol. Cybern. 84, 153-172. doi: 10.1007/s004220000209

Sun, H., Luo, L., Yuan, X., Zhang, L., He, Y., Yao, S., et al. (2018). Regional homogeneity and functional connectivity patterns in major depressive disorder, cognitive vulnerability to depression and healthy subjects. J. Affect. Disord. 235, 229-235. doi: 10.1016/j.jad.2018.04.061

Tu, X., Wang, J., Liu, X., and Zheng, J. (2018). Aberrant regional brain activities in alcohol dependence: a functional magnetic resonance imaging study. Neuropsychiatr. Dis. Treat. 14, 847-853. doi: 10.2147/NDT.S158221

Ward, L. M. (2003). Synchronous neural oscillations and cognitive processes. Trends Cogn. Sci. 7, 553-559. doi: 10.1016/j.tics.2003.10.012

Zapp, D., Loos, D., Feucht, N., Ramin, K., Tamer, T., Lukas, R., et al. (2018). Microbial keratitis-induced endophthalmitis: incidence, symptoms, therapy, visual prognosis and outcomes. BMC Ophthalmol. 18:112. doi: 10.1186/s12886018-0777-3

Zhang, P., Li, Y., Fan, F., Li, C., Luo, X., Yang, F., et al. (2018). Resting-state brain activity changes associated with tardive dyskinesia in patients with schizophrenia: fractional amplitude of low-frequency fluctuation decreased in the occipital lobe. Neuroscience 385, 237-245. doi: 10.1016/j.neuroscience.2018. 06.014

Zhang, Y., Yang, Y., Wang, Z., Bian, R., Jiang, W., Yin, Y., et al. (2018). Altered regional cerebral blood flow of right cerebellum posterior lobe in asthmatic patients with or without depressive symptoms. Front. Psychiatry. 9:225. doi: 10.3389/fpsyt.2018.00225

Zheng, J., Chen, Y., Chen, H., Jiang, L., Bo, F., Feng, Y., et al. (2018). Disrupted spontaneous neural activity related to cognitive impairment in postpartum women. Front. Psychol. 9:624. doi: 10.3389/fpsyg.2018. 00624

Zheng, W., Su, Z., Liu, X., Zhang, H., Han, Y., Song, H., et al. (2018). Modulation of functional activity and connectivity by acupuncture in patients with Alzheimer disease as measured by restingstate fMRI. PLoS One 13:e196933. doi: 10.1371/journal.pone.019 6933

Conflict of Interest Statement: The authors declare that the research was conducted in the absence of any commercial or financial relationships that could be construed as a potential conflict of interest.

Copyright (C) $2019 X u, L i u$, Tan, Su, Xiang, Wu, Li, Lin, Xu, Min, Liu, Gao and Shao. This is an open-access article distributed under the terms of the Creative Commons Attribution License (CC BY). The use, distribution or reproduction in other forums is permitted, provided the original author(s) and the copyright owner(s) are credited and that the original publication in this journal is cited, in accordance with accepted academic practice. No use, distribution or reproduction is permitted which does not comply with these terms. 